\title{
Measuring Rapid Time-scale Reaction Kinetics Using Isothermal Titration Calorimetry
}

\author{
Justin M. Di Trani $\uparrow$, Nicolas Moitessier $\dagger$, and Anthony K. Mittermaier*, $\dagger$ \\ $†$ Department of Chemistry, McGill University, H3A 0B8 Montreal, Canada \\ *Email: anthony.mittermaier@mcgill.ca, Fax: 514-398-3797
}

\begin{abstract}
Isothermal titration calorimetry (ITC) is a powerful tool for acquiring both thermodynamic and kinetic data for biological interactions including molecular recognition and enzymatic catalysis. ITCbased kinetics measurements typically focus on reactions taking place over long timescales (tens of minutes or hours) in order to avoid complications due to the finite length of time needed detect heat flow in the calorimeter cell. While progress has been made towards analyzing more rapid reaction kinetics by ITC, the capabilities and limitations of this approach have not been thoroughly tested to date. Here, we report that the time resolution of commercial instruments is on the order of 0.2 seconds or less. We successfully performed rapid ITC kinetics assays with durations of just tens of seconds using the enzyme trypsin. This is substantially shorter than previous ITC enzyme measurements. However we noticed that for short reaction durations, standard assumptions regarding the ITC instrument response led to significant deviations between calculated and measured ITC peak shapes. To address this issue, we developed an ITC empirical response model (ITC-ERM) that quantitatively reproduces ITC peak shapes for all reaction durations. Applying the ITC-ERM approach to another enzyme (prolyl oligopeptidase), we unexpectedly discovered non-Michaelis-Menten kinetics in short time-scale measurements that are absent in more typical long time-scale experiments and are obscured in short time-scale experiments when standard assumptions regarding the instrument response are made. This highlights the potential of ITC measurements of rapid timescale kinetics in conjunction with the ITC-ERM approach to shed new light on biological dynamics.
\end{abstract}

Isothermal titration calorimetry (ITC) is a powerful tool for determining thermodynamic and kinetic parameters for a range of biological reactions.[1,2] The instrument makes periodic injections of a macromolecule, ligand, or substrate solution into a sample cell containing a molecule of interest while recording how much power must be supplied to maintain a constant temperature. Exothermic reactions result in transient decreases in power, relative to the baseline, while endothermic reactions result in transient increases in the power supplied to the sample cell. ITC is commonly used to measure binding thermodynamics. In these applications, each peak in the ITC isotherm is integrated to yield the total amount of heat released or absorbed during and following each injection. The resultant series of integrated heats can be fit to variety of equations to yield the affinity, enthalpy, entropy, and stoichiometry of binding interactions.[3] ITC can also be used to measure reaction kinetics. This is accomplished by analyzing the shapes of the peaks themselves, which are related to the timescale of heat generation during and following each injection. Rapid reactions lead to sharp ITC peaks that are complete in seconds or tens of seconds while slow reactions lead to broad peaks that may be minutes or even hours in length.[4, 5] Quantitative ITC peak shape analysis has been used to characterize the kinetics of a wide variety of reactions including the chemical reactions of small molecules,[6] enzyme catalysis, [7-17] and biomolecular binding. [17-22]

ITC offers several advantages over other experimental kinetics methods such as surface plasmon resonance,[23] UV-vis spectrophotometry,[24-26] and nuclear magnetic resonance spectroscopy.[27] It can be performed entirely in solution under physiological conditions, does not require spectroscopicallyactive (eg. fluorescent) molecules, it is compatible with spectroscopically opaque solutions, [7] and can be applied to relatively dilute samples.[4] Despite its long history and technical advantages, kinetic applications of ITC remain fairly rare. A potential factor is the challenge of accurately modeling ITC peak shapes. This requires accounting for the various mechanical, chemical, and electrical processes that are involved in the measurement of an ITC isotherm. In practice, an instantaneous release of heat in the ITC 
cell is detected over a period of seconds or tens of seconds. When reactions take place on similar time scales to these processes, it is challenging to separate the kinetics of the reaction from the kinetics of the instrument response itself.[11, 19] In contrast, for slower reactions taking place on the timescale of minutes to hours, the kinetics of the instrument response can essentially be ignored. Nevertheless, many interesting and biologically important reactions take place on the seconds timescale. Progress has been made towards modelling the ITC instrument response and in measuring rapid reaction kinetics by ITC.[19] However to date the accuracy of the instrument response models has not been thoroughly tested, nor has the upper limit for reaction rates in ITC experiments been systematically explored.

In this study, we have used a combination of electric heater pulses, $\mathrm{Ca}^{2+} /$ EDTA binding experiments, and enzyme assays with trypsin and prolyl oligopeptidase to map out the capabilities and limitations of rapid kinetics experiments using common benchtop ITC instruments, focusing mainly, but not exclusively, on the VP-ITC produced by MicroCal/Malvern. We found that this instrument is capable of extracting reaction times with sub-second accuracy (as is the MicroCal ITC-200 and the TA Instruments Nano ITC). Motivated by this result, we performed trypsin enzymatic reactions in which all of the injected substrate was exhausted in under a minute, which is more rapid than previous ITC enzyme assays by about a factor of 20. Using a previously-published kinetic ITC analysis technique,[9] we extracted enzyme parameters in agreement with literature values. However, the modeled ITC peak shapes were not well reproduced, potentially obscuring useful kinetic information. We therefore developed an ITC Empirical Response Model (ITC-ERM), which accurately reproduces both ITC peak shapes and kinetic parameters. Applying the ITC-ERM approach to rapid reactions of prolyl oligopeptidase, we observed surprising non-MichaelisMenten kinetics that are completely obscured by previous ITC and spectroscopic analysis methods.

\section{EXPERIMENTAL SECTION}

ITC Experiment Conditions. All ITC experiments were performed using a MicroCal VP-ITC in highfeedback mode with a 1 second signal-averaging window and stirring rate of $806 \mathrm{rpm}$, unless otherwise specified. Pre-injection delays of 500 seconds were employed to allow baselines to fully stabilize.

Electrical Heater Pulses. Electrical heater pulses were executed using the 'pulse-off' command in the VPViewer2000 Thermostat/Calibration tab during a 2000s pre-titration delay with stirring. The peaks produced by the electric heater pulses were normalized by the total area of the peak (equation 8).

Enzymes and Substrate preparation. Trypsin (EC 3.4.21.4) was purchased in the form of (TrypZeanTM) from Sigma-Aldrich. The lyophilized powder was dissolved into $200 \mathrm{mM}$ Tris-HCl, pH 8.0, 50mM CaCl , and $0.2 \%$ PEG-8000 buffer. The concentration of Trypsin was measured using the extinction coefficient at $280 \mathrm{~nm}\left(\mathcal{E}=30057 \mathrm{M}^{-1} \mathrm{~cm}^{-1}\right)$.[28] The substrate $\mathrm{N} \alpha$-Benzoyl-L-arginine ethyl ester (EC 220-157-0) was purchased from Sigma-Aldrich and dissolved into the same buffer.

Human Prolyl oligopeptidase was purified as described previously[29] and dialyzed in a buffer containing $20 \mathrm{mM}$ sodium phosphate $\mathrm{pH} 8,150 \mathrm{mM}$ sodium chloride, and $10 \%(\mathrm{w} / \mathrm{v})$ glycerol, prior to flash freezing in $300 \mathrm{uL}$ aliquots at $\approx 4 \mu \mathrm{M}$ in liquid nitrogen and storing at $-80^{\circ} \mathrm{C}$. Kinetic experiments were carried out with freshly thawed POP in the same buffer (above) with bovine serum albumin (BSA, $0.5 \mathrm{mg} / \mathrm{mL}$ ) added to help stabilize POP. The substrate thyrotropin releasing hormone (TRH) was purchased from BACHEM international (product H-4915) and dissolved into the same buffer as POP for kinetics experiments.

$\mathrm{Ca}^{2+}$ EDTA titrations. All $\mathrm{Ca}^{2+}$-EDTA measurements in the main text (except saturation experiments) were performed at $25^{\circ} \mathrm{C}$ in $10 \mathrm{mM}$ MES (2-( $N$-morpholino)ethanesulfonic acid) buffer $\mathrm{pH}$ 6. The $\mathrm{Ca}^{2+}-$ EDTA saturation titrations were performed at $25^{\circ} \mathrm{C}$ in $20 \mathrm{mM}$ PIPES (piperazine- $N, N^{\prime}$-bis (2ethanesulfonic acid)) buffer $\mathrm{pH}$ 7. The peaks generated by the $\mathrm{Ca}^{2+}$-EDTA injections were normalized (equation 8). 
Enzyme Kinetics Fitting Scripts. All fitting was performed in MATLAB. Differential equations shown below (equations 1-4) describing Michaelis-Menten kinetics were integrated numerically using Euler's method in $0.01 \mathrm{~s}$ integration steps (dt in equations 1-4) according to:

$$
\begin{gathered}
\frac{d[P]_{t}}{d t}=\frac{k_{\text {cat }} *[E] *[S]_{t}}{K_{m}+[S]_{t}} \\
{[P]_{t+d t}=[P]_{t}+\frac{d[P]_{t}}{d t} * d t} \\
\frac{d[S]_{t}}{d t}=-\frac{k_{c a t} *[E] *[S]_{t}}{K_{m}+[S]_{t}}+\frac{\text { Rate }_{\text {inj }}}{V_{\text {cell }}} \\
d[S]_{t+d t}=[S]_{t}+\frac{d[S]_{t}}{d t} * d t
\end{gathered}
$$

where $k_{c a t}$ and $K_{m}$ are the catalytic rate and the Michaelis constant respectively, $[E]$ is the total concentration of enzyme in the cell and $[S]_{t}$ and $[P]_{t}$ are the concentrations of substrate and product at time $=\mathrm{t}$. Rate $_{i n j}$ is the rate of the injection $\left(\mathrm{mole} \mathrm{s}^{-1}\right)$ and $V_{\text {cell }}$ is the total volume of the reaction cell. Note that on MicroCal instruments the beginnings of injections are indicated with an '@' sign in the raw data file. On the TA Nano instrument, injection start times are specified in advanced. This was verified visually by the user. The instantaneous heat $\mathrm{h}(\mathrm{t})$ is calculated using the enthalpy of the reaction $\Delta H_{\text {react }}$ and the total volume of the cell (equation 5).

$$
h(t)=\Delta H_{\text {react }} * V_{\text {cell }} * \frac{d[P]_{t}}{d t}
$$

The instantaneous heat curve is numerically convoluted with the instrument response function according to:

$$
g(t)=h(t) \otimes f(t)
$$

where $f(t)$ is either a mono-exponential response function or the empirical response function (obtained from reference injections, see Results Section), $g(t)$ is the resulting calculated signal, and the convolution is defined according to

$$
h(t) \otimes f(t)=\int_{o}^{t} f(\tau) g(t-\tau) d \tau
$$

The calculated instrument output, $g(t)$, was digitally resampled in second intervals to match the experimental calorimeter output and normalized by the total area of the peak using trapezoidal integration according to

$$
g_{\text {norm }}\left(t_{m}\right)=\frac{g\left(t_{m}\right)}{\sum_{n=0}^{N}\left[g\left(t_{n}+1\right)+g\left(t_{n}\right)\right] * d t / 2}
$$

where $\mathrm{N}$ is the total number of time points, $\mathrm{tn}$ and tm are the $\mathrm{nth}$ and $\mathrm{mth}$ timepoint, $\mathrm{dt}$ is the time increment ( 1 second in all cases here) and $g_{\text {norm }}$ is the normalized peak. Enzyme kinetic parameters as well as a scaling factor for the modelled curve $\mathrm{Ng}$ were fit by minimizing the target function (equation 5)

$$
R S S=\sum_{n=0}^{N}\left(N_{g} * g_{\text {norm }}\left(t_{n}\right)-b_{\text {norm }}\left(t_{n}\right)\right)^{2}
$$


where RSS is the residual sum of squared differences, $b_{\text {norm }}(t)$ is experimental data which has been normalized according to equation 8.

All enzyme experiments were performed in triplicate. Errors of fits as well as parameter correlation coefficients were calculated using the variance-covariance matrix (see supporting information).

\section{RESULTS}

Background. There are several related physical and chemical processes that must be considered when calculating the time-dependent ITC signal. Firstly, there is the user-selected finite injection time, during which some of the contents of the syringe are introduced to the sample cell, typically on the order of 1500 seconds. Subsequently, during the pre-reaction mixing time, the injected solution is mixed homogeneously throughout the cell.[19] As the contents of the injection and sample cell are mixed, they can interact according to their intrinsic chemical kinetics, $[4,19]$ leading to the release or absorption of heat and the local heating or cooling of the solution at the site of reaction. This is followed by a heat transfer delay, the length of time necessary for the solid phase thermocouple to detect the small change in sample cell temperature, relative to the reference cell[30-33]. In an ITC feedback calorimeter this temperature difference activates the electronic response feedback circuit which causes a change in the amount of power being supplied to the sample cell, driving the temperature gradient back to zero.[31] Together, these processes determine the ultimate shape of the instrument output, i.e. the time-dependent signal (usually in $\mu \mathrm{cal} / \mathrm{s}$ ) that is recorded by the ITC. The preceding processes can all potentially contribute to the shape of a peak, however, the relative contribution and importance of each step has not been the subject of much attention to date.

Typically, the finite injection time, pre-reaction mixing, and chemical kinetic processes are combined to calculate the time-dependent rate at which heat is generated in the ITC cell, which we refer to as the instantaneous heat or $h(t)$ (see Supporting Information section).[19] The post-reaction heat transfer and electronic response are usually modelled as a single function, which we refer to as $f(t)$. In most applications published to date, $\mathrm{f}(\mathrm{t})$ has been expressed as a single- or bi-exponential decaying function. [19, 20, 32, 34-36] When $f(t)$ is modelled as single-exponential, $f(t)=\exp \{-t / \tau i\}$

, the constant $\tau \mathrm{i}$ is referred to as the time constant of the ITC instrument and is typically taken to be on the order of 5 to 15 seconds.[19] The instrument output, $g(t)$, is then calculated as the convolution of the instantaneous heat and the instrument response, $\mathrm{g}(\mathrm{t})=\mathrm{h}(\mathrm{t}) \otimes \mathrm{f}(\mathrm{t})$ (See Methods Section). Physically, this implies that an instantaneous burst of heat in the calorimeter cell produces a signal with the shape of the response function, i.e. if $h(t)$ is the Dirac delta function, then $g(t)=f(t)$.

Rapid injection Experiments. In order to test the ITC instrument outputs resulting from known inputs, we used EDTA and $\mathrm{Ca}^{2+}$ as a model host/guest binding reaction. This system has the advantage of very strong affinity $(\mathrm{KD} \approx 0.180 \mu \mathrm{M})$ [37] providing tight control over reaction stoichiometry, high reaction enthalpy $\left(\Delta \mathrm{H} \approx-3.5 \mathrm{kcal} \mathrm{mol}^{-1}\right)$ giving large heat signals, and extremely rapid association rates $\left(\mathrm{kon} \approx 5 \times 10^{6} \mathrm{M}^{-1} \mathrm{~s}^{-}\right.$ $\left.{ }^{1}\right),[37]$ meaning that the shapes of ITC peaks are dominated by factors other than the reaction kinetics, as desired for characterizing the instrument response. The pseudo first order time constant ( $\tau$ react $=$ (kon[EDTA])-1) for all $\mathrm{Ca}^{2+} /$ EDTA reactions recorded in this study are less than $2.5 \mathrm{~ms}$. We performed a series of injections of $\mathrm{Ca}^{2+}$ into EDTA lasting between 0.2 and 5 seconds. During and following each injection we observed a deflection in the power due to the heat released by the $\mathrm{Ca}^{2+} / \mathrm{EDTA}$ binding reaction, as shown in Figure 1. Although the heat signals are tens of seconds in length, the longer injections nevertheless give rise to peaks that are noticeably wider; the injections differ from each other by only 1 second but produce peaks that are clearly distinct. This demonstrates that a typical ITC instrument has sub-second time-resolution, as required for measuring rapid kinetic processes. 


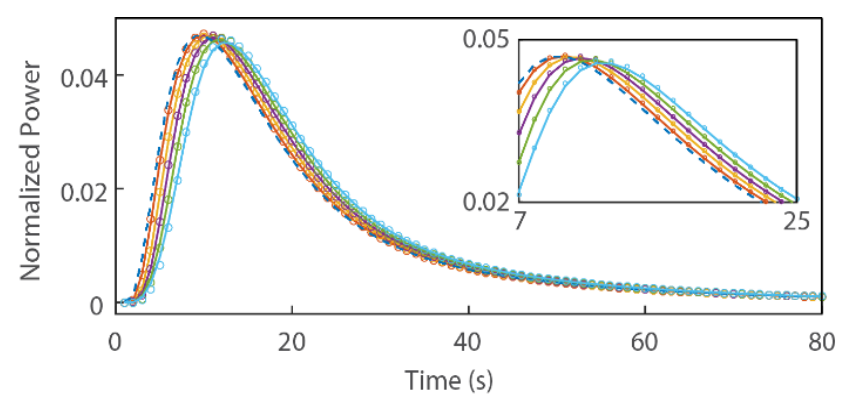

Figure 1. Rapid injections into EDTA using the VP-ITC. CaCl2 $(18 \mathrm{mM}$ in syringe) was injected $(1 \mu \mathrm{L}$ injections) into the cell containing $1.8 \mathrm{mM}$ EDTA. Area-normalized peaks (averages of 10 replicates) are shown for $0.2 \mathrm{~s}$ (dashed blue line), 1s (orange circles), $2 \mathrm{~s}$ (yellow circles), 3s (purple circles), 4s (green circles) and $5 \mathrm{~s}$ (cyan circles) injections. The solid lines show the fit to the experimental peaks using the ERM approach (see below) while floating the injection length. Extracted lengths for the injections are plotted in Figure S2A.

Rapid Time-Scale Enzyme Kinetics. The ITC traces obtained from injections of $\mathrm{Ca}^{2+}$ into EDTA, described above, suggest that short bursts of heat are detected over approximately 40 seconds by the VPITC instrument. We therefore aimed to test whether enzymatic reactions occurring on similar timescales can be accurately characterized by this calorimeter. We selected the well-studied enzyme trypsin and the substrate Na-Benzoyl-L-arginine ethyl ester hydrochloride (BAEE) as a model system. Initial experiments were performed by injecting BAEE into the cell containing relatively low concentrations of trypsin, on the order of $10 \mathrm{nM}$ (Figure 2A). The observed heat signal from the instrument output is about 150 seconds in duration, indicating that the total duration of the enzyme reaction is slightly greater than 100 seconds, as the heat generated by the enzyme is detected with up to a roughly 50 second delay. The data were fit using a recently-developed approach in which the instantaneous heat, $h(t)$, is calculated according to the Michaelis-Menten equation, the instrument response, $f(t)$, is assumed to be singleexponential, and the enzyme kinetic parameters are extracted while simultaneously fitting an effective value for the instrument response time, $\tau i$.[9] In what follows, we refer to this as the variable- $\tau$ approach. The modelled curve closely follows the experimental data and the fitted parameters $(\mathrm{Km}=3.42 \pm 0.08$ $\mu \mathrm{M}$; kcat $\left.=20.0 \pm 0.3 \mathrm{~s}^{-1} ; \tau_{i}=23.9 \pm 0.34 \mathrm{~s}\right)$ are in good agreement with previously reported values $(\mathrm{Km}$ $=4 \mu \mathrm{M}$; $\left.\mathrm{kcat}=15 \mathrm{~s}^{-1}\right)$. [4, 38] Next, more rapid transients were produced using higher concentrations of enzyme, on the order of $60 \mathrm{nM}$ (Figure 2B). In this case, the instrument signal is roughly 70 seconds length, which is only 20 seconds longer in duration than the signal produced by a rapid injection of $\mathrm{Ca}^{2+}$ into EDTA. In other words, this enzymatic reaction occurs on a timescale equal to or less than the instrument response time. Once again, the data were fit using the variable- $\tau$ procedure. The extracted parameters $\left(\mathrm{Km}=4.24 \pm 0.05 \mu \mathrm{M}\right.$; kcat $\left.=16.7 \pm 0.1 \mathrm{~s}^{-1} ; \tau_{i}=16.4 \pm 0.16 \mathrm{~s}\right)$ agreed well with reported values, however the modelled curve deviated substantially from the experimental data. Trypsin has been shown to follow Michaelis-Menten kinetics in numerous studies. This strongly suggests that the variable$\tau$ procedure used to model the ITC response yields distorted peak shapes, at least for rapid reactions, potentially obscuring important kinetic information.
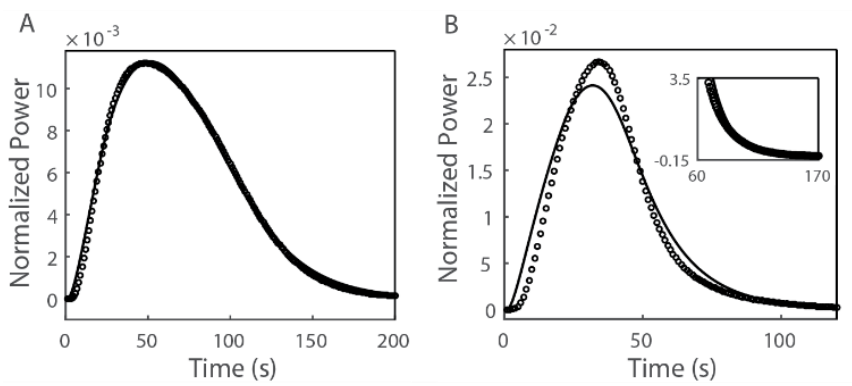
Figure 2. Single Injection Enzyme Kinetics for Trypsin using the VP-ITC. (A) Single injection $(30 \mu \mathrm{L}$ over $20 \mathrm{~s})$ of BEAA ( $400 \mu \mathrm{M}$ in syringe) into the cell containing trypsin 10nM (open circles). Best fit according to the variable- $\tau$ approach.[11] (solid line). (B) Single injection of BEAA (1100 $\mu \mathrm{M}$ in syringe) into the cell containing trypsin $60 \mathrm{nM}$ (open circles). Best fit according to the variable- $\tau$ approach.[11] (solid line).

Factors affecting ITC peak shapes. In order to develop an analytical approach that more closely reproduces ITC kinetic peak shapes, we re-examined the assumptions of the variable- $\tau$ method used above to analyze trypsin kinetics. These are 1) that pre-reaction mixing is effectively instantaneous and 2) that the postreaction response of the calorimeter $(\mathrm{f}(\mathrm{t})$ in equation 7$)$ is a single exponential decaying function. Inspection of the $\mathrm{Ca}^{2+} /$ EDTA traces in Figure 1 reveals that either one or both of these assumptions must be false. A short injection $(0.2 \mathrm{~s})$ produces a heat signal that increases up to the 9 -second mark. For instantaneous mixing with a single-exponential response function, the maximum heat flow is necessarily at the end of the injection period. In this case, the maximum heat flow is obtained 9 seconds after the end of the injection.

One possible explanation for this delayed response is that it takes several seconds for the injected $\mathrm{Ca}^{2+}$ to mix with the EDTA in the cell. Note that the rate constant for this reaction $\left(5 \times 10^{6} \mathrm{M}^{-1} \mathrm{~s}^{-1}\right)$ [37] is sufficiently large that it may be assumed that $\mathrm{Ca}^{2+}$ and EDTA react instantaneously once mixed. We tested the mixing time by performing a series of $\mathrm{Ca}^{2+}$ injections into a highly concentrated solution of EDTA (Figure 3), with a c-value ([EDTA]/KD) of roughly 500. In the second of these injections (the first injection is ignored due to pre-titration diffusion from the syringe), there is sufficient EDTA to fully react with injected $\mathrm{Ca}^{2+}$ in $7 \mu \mathrm{l}$ of the cell solution, i.e. just $5 \%$ of the total cell volume. In this case, one would expect that sufficient mixing of the two components would occur very rapidly. With subsequent injections, as free EDTA is converted to the bound form, the amount of mixing required for each injection increases. By the 20th injection, the $\mathrm{Ca}^{2+}$ must fully mix with at least $630 \mu \mathrm{l}$ of the partly-saturated EDTA solution ( $45 \%$ of the cell volume) in order to react to completion. In this case we would expect that sufficient mixing would require more time than for the first injection. Notably, all $\mathrm{Ca}^{2+}$ injections, from the second to the 20th give superimposable peak shapes, although the amount of mixing required for each injection can vary by up to about 90 -fold. This strongly suggests that pre-reaction mixing is not ratelimiting for generation and detection of heat in the ITC under these conditions. We note that the signal from the $21^{\text {st }} \mathrm{Ca}^{2+}$ injection is substantially broader than that of the previous injections. In this case, mixing with at least $79.5 \%$ of the cell volume is required to reach completion, and it seems likely that in this case, the pre-reaction mixing step becomes at least partially rate-limiting. Nevertheless, the assumption of instantaneous mixing appears to be valid for a broad range of free EDTA concentrations.

Another possible explanation for the observed ITC peak shapes is that the post-reaction heat transfer and electronic response do not combine to give a single exponential response function. In order to examine these steps more closely, we measured the ITC signals resulting from short impulses of the electrical heater used to calibrate the sample cell. Here again, we observed heat signals that continued to build for roughly 9 seconds following the end of the heater pulse, despite the fact that no mixing of reactants is required (Figure S4). We repeated these experiments with the water-filled syringe present in the cell rotating at different stirring speeds. Notably, we observed broader peaks at slower stirring speeds. Furthermore, the stirring speed dependences of ITC peaks obtained from heater pulses and $\mathrm{Ca}^{2+} / \mathrm{EDTA}^{2}$ injections are very similar. This strongly suggests that stirring affects the post-reaction heat transfer step, and that this together with the electronic feedback mechanism largely governs the ITC peak shapes measured here. 


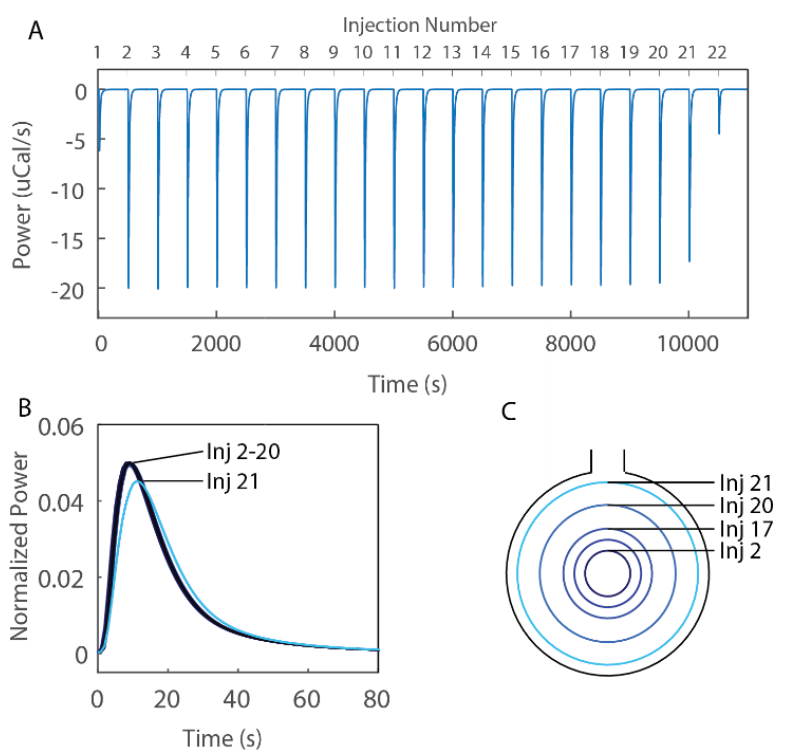

Figure 3. $\mathrm{Ca}^{2+}$ EDTA saturation experiments using the VP-ITC. (A) Titration of $\mathrm{CaCl}^{2} 11 \mathrm{mM}$ into EDTA $1.8 \mathrm{mM}$ until saturation form a single experiment. (B) Overlay of area-normalized peaks 2-20 and 21. (C) Representation of coin shaped cell (outer ring with neck). The area of each inner disk is proportional to the volume of cell solution which contains enough unbound EDTA to bind all $\mathrm{CaCl}^{2}$ provided in that injection. The first injection small $(5 \mu \mathrm{L}$ over $10 \mathrm{~s})$ is not shown in panel $\mathrm{B}$ or $\mathrm{C}$ due to pre titration diffusion form the tip of the syringe into the cell all subsequent injections are $10 \mu \mathrm{L}$ over $2 \mathrm{~s}$.

Empirical Response Model. We have a developed an approach for analyzing ITC peak shapes based on an empirical response model (ERM). The approach treats pre-reaction mixing as effectively instantaneous, as outlined above, and subsumes all post-reaction heat transfer and electronic response into a single empirical response function, $f(t)$, which obeys the convolution relationship in equation 7 . We took advantage of the property of convolutions, wherein an infinitely short burst of heat in the calorimeter cell $(\mathrm{h}(\mathrm{t})=\delta(0))$ produces $\mathrm{g}(\mathrm{t})$ as the instrument output. Here, we approximated an instantaneous burst of heat with a 0.1 to 0.5 second injection of $\mathrm{Ca}^{2+}$ into EDTA, and took the resulting peak as the empirical response function, $\mathrm{f}(\mathrm{t})$. ITC peak shapes were then simulated by numerically convoluting the instantaneous heat profile $(\mathrm{h}(\mathrm{t}))$ with the empirical response function $(\mathrm{f}(\mathrm{t}))$ thus obtained. We tested the ITC-ERM by fitting $\mathrm{Ca}^{2+} /$ EDTA injections of varying durations and comparing the known injection lengths with those extracted from the peak shapes. This approach reproduced the experimental ITC peaks with remarkable fidelity (Figure 1), and the extracted injection lengths closely match the set values with a root-mean-square deviation of only $0.2 \mathrm{~s}$ (Figure S2). We then repeated the kinetic analysis of trypsin using the ITC-ERM approach and assuming Michaelis-Menten enzyme kinetics, obtaining very close agreement between the simulated and experimental peak shapes (Figure 4B), and kinetic parameters that match the literature values $\left(\mathrm{Km}=2.848 \pm 0.001 \mu \mathrm{M}\right.$; kcat $\left.=16.69 \pm 0.02 \mathrm{~s}^{-1}\right)$. This is in contrast with the variable- $\tau$ method (Figure $2 \mathrm{~B}$ ), where simulated ITC peak shapes show large systematic deviations from the experimental data. These results give us confidence that the ITC-ERM approach quantitatively reproduces ITC peaks of arbitrarily short duration with a high degree of accuracy.
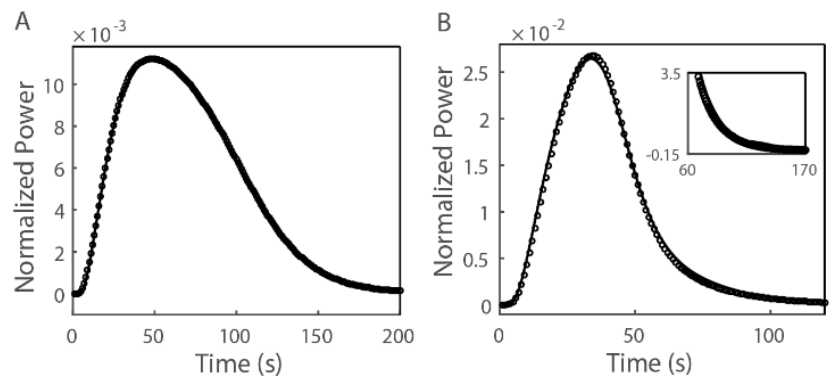
Figure 4. Single Injection Enzyme Kinetics for Trypsin using the VP-ITC. (A) Single injection of BEAA (400 $\mu \mathrm{M}$ in syringe) into the cell containing trypsin 10nM (open circles). Curve produced using ITC-ERM method. (solid line). (B) Single injection of BEAA $(1100 \mu \mathrm{M}$ in syringe) into the cell containing trypsin 60nM (open circles). Curve produced using ITC-ERM method. (solid line).

Prolyl Oligopeptidase. Prolyl oligopeptidase (POP), is a post-proline cleaving enzyme implicated in cancer and neurodegenerative disorders.[39, 40] POP cleaves thyrotropin-releasing hormone (TRH), a modified tri-peptide with the sequence (pyro)Glu-His-Pro- $\mathrm{NH}_{2}$, among other substrates. ITC traces obtained from injections of TRH into the reaction cell containing varying concentrations of POP are shown in Figure 5. The ITC-ERM approach was used to fit each transient using the Michaelis-Menten equation. At low enzyme concentrations, the calculated peak shapes agree well with the experimental data (Figure 5A), as seen for trypsin, above. However, at higher enzyme concentrations, the peak shapes are no longer well fit by this model Instead, the data agree with an empirical formula, previously used to describe non-Michaelis-Menten enzymes with cooperative substrate binding,[41] according to:

$$
\frac{d[P]_{t}}{d t}=\frac{k_{c a t} *[E] *[S]^{n}}{K_{m}+[S]^{n}}
$$

where values of $n>1$ correspond to positive cooperativity and $n<1$ indicate negative cooperativity. This behavior is fundamentally different from that of trypsin, whose ITC peak shapes agree closely with classical Michaelis-Menten kinetics at both low and high enzyme concentrations. In the case of POP, values of the cooperativity coefficient vary from $n=1.13$ with $[P O P]=136 \mathrm{nM}$ to $n=2.37$ with $[\mathrm{POP}]=1.98$ $\mu \mathrm{M}$ (see Table S2). Interestingly, the extracted values of $\mathrm{n}$ appear to follow an empirically linear dependence on enzyme concentration. Extrapolation to the y-intercept is short, and produces a value of $\mathrm{n}=1.07$. This suggests that at vanishing low enzyme concentrations, POP obeys Michaelis Menten kinetics almost exactly, while at higher enzyme concentrations, kinetics are increasingly cooperative, implicating enzyme self-association in solution in this behavior. To our knowledge, such a concentration-dependent shift in enzyme cooperativity has not been previously reported for POP. The underlying physical mechanism and potential physiological role are not known, and are the subject of ongoing investigation in our laboratory. Interestingly, the cooperative kinetic behavior of POP disappears at the low enzyme concentrations $(\approx 3 \mathrm{nM})$ that have been used in previous spectroscopic assays with POP[42] and multiple injection experiments (Figure S5). Furthermore, when the rapid enzyme kinetic data is modelled using an exponential response function with the variable- $\tau$ approach, the inherent systematic distortion of the peak shapes (Figure 2B) entirely masks the non-Michaelis-Menten behavior. In other words, rapid singleinjection enzyme kinetic ITC assays together with ITC-ERM analysis represent a uniquely powerful approach for unravelling non-classical enzyme kinetics. 

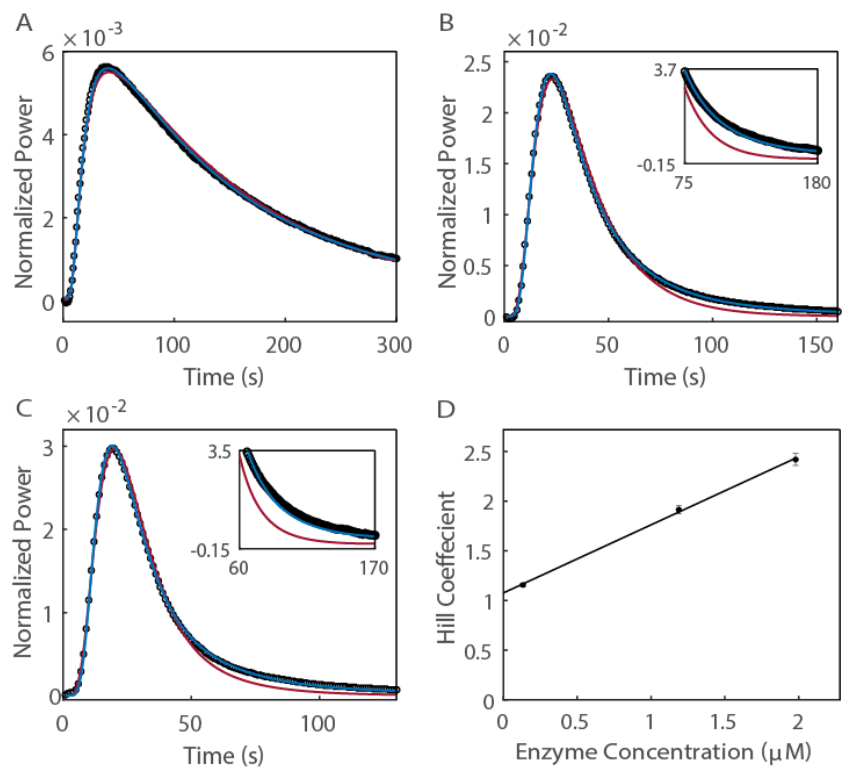

Figure 5. Single Injection Enzyme Kinetics for POP using the VP-ITC. All three panels show data for a single injection $(20 \mu \mathrm{L}$ over $6 \mathrm{~s})$ of TRH $(690 \mu \mathrm{M}$ in syringe) made into a cell containing $132 \mathrm{nM}$ POP (A), $1.8 \mu \mathrm{M}$ POP (B), and $2.37 \mu \mathrm{M}(\mathrm{C})$ (open circles). Fits using the ITC-ERM approach and MichaelisMenten kinetics (equation 1) and cooperative kinetics (equation 10) are shown in red and blue lines, respectively at each concentration. Resulting Hill coefficients (n) plotted against enzyme concentration with line obtained by linear regression (C).

Maximum Reaction Rates. Chemical reactions that take place on roughly the same timescale as the instrument response can be quantified by ITC-ERM, while those that occur much more rapidly (eg. short $\mathrm{Ca}^{2+} /$ EDTA injections) are indistinguishable from instantaneous bursts of heat and yield little or no kinetic information. In order to estimate rough upper rate limits for this technique, we performed a series of Monte Carlo calculations in which molecular binding and enzyme catalytic data were computed with simulated random errors. The synthetic data were analyzed using the ITC-ERM method and the fitted kinetic parameters were compared to the ones used to generate the data sets. For molecular recognition, we fixed $\mathrm{K}_{\mathrm{D}}$ and $\Delta \mathrm{H}$ to the $\mathrm{Ca}^{2+} /$ EDTA-derived values $(0.180 \mu \mathrm{M}$ and $-3.5 \mathrm{kcal} / \mathrm{mol}$, respectively) and varied $\mathrm{k}_{\text {on }}$ between $10^{4}$ and $10^{6} \mathrm{M}^{-1} \mathrm{~s}^{-1}$. The agreement between the true and fitted kon values was excellent for the slower on-rates, but increased rapidly after $1 \times 10^{5} \mathrm{M}^{-1} \mathrm{~s}^{-1}$, reaching more than $50 \%$ error after about $4 \times 10^{5} \mathrm{M}^{-1} \mathrm{~s}^{-1}$. Association rates faster than this lead to signals that are essentially indistinguishable from instantaneous bursts of heat. Note that the $\mathrm{k}_{\text {on }}$ values for $\mathrm{Ca}^{2+} /$ EDTA are about 10 times this value. Thus $\mathrm{k}_{\text {on }}=10^{5} \mathrm{M}^{-1} \mathrm{~s}^{-1}$ represents an approximate upper limit for association rates, under these conditions. Interestingly this is slightly faster than the ITC-derived rate constant of $3 \times 10^{4} \mathrm{M}^{-1} \mathrm{~s}^{-1}$ reported by the Dumas lab for the TPP riboswitch.[19] For the enzyme simulations, we fixed kcat and Km to the trypsinderived values (15 s $\mathrm{s}^{-1}$ and $4 \mu \mathrm{M}$, respectively) and varied the total enzyme concentration between 5 and $1000 \mathrm{nM}$. The agreement between the true and fitted $\mathrm{k}_{\mathrm{cat}}$ and $\mathrm{K}_{\mathrm{m}}$ values was excellent for the lower enzyme concentrations, where heat is generated more slowly. In contrast, for concentrations greater than about $400 \mathrm{nM}\left(\mathrm{V}_{\max }>6 \mu \mathrm{M} \mathrm{s}^{-1}\right)$, the errors in the extracted $\mathrm{k}_{\text {cat }}$ and $\mathrm{K}_{\mathrm{m}}$ values were larger than $20 \%$ and $50 \%$, respectively, marking the approximate upper limit for this technique under these conditions.

Application to Other ITC Instruments. In order to test the generality of the ITC-ERM approach, we repeated $\mathrm{Ca}^{2+}$ EDTA reference experiments using the MicroCal ITC-200, which has a cell size approximately seven-fold smaller than the VP-ITC, and the Nano ITC from TA instruments, which has a cell roughly the same size as that of the ITC-200, but cylindrical in shape, in contrast to the coin-shaped cells employed in the MicroCal instruments. In all cases, injection lengths varying between 1 and 5 seconds are clearly distinguishable, indicating that these instruments also have sub-second time resolution (Figure S1). The variable-length injections were then fitted using the ITC-ERM approach, as described 
above for the VP-ITC. Interestingly, for both the ITC-200 and the Nano ITC, the fits deviated systematically, such that injection lengths were consistently under-estimated (Figure S2). We suspected that the programmed $0.2 \mathrm{~s}$ pulses were not serving as an accurate models of the instrument response function, $\mathrm{f}(\mathrm{t})$. In order to circumvent this problem, we reanalyzed the data using a longer (1 second) injection to model the instrument response and adjusting the experimental data accordingly. Briefly, we define a modified response function, $\mathrm{f}^{\prime}(\mathrm{t})$ as the area-normalized instrument output following a 1 second injection, and the adjusted data, $g^{\prime}(t)$ to be the instrument output numerically convoluted with a 1 second square pulse, $\mathrm{s}(\mathrm{t})=1,0 \leq t \leq 1$ and $\mathrm{s}(\mathrm{t})=0, \mathrm{t}<0, \mathrm{t}>1$. The modified response function can be used to fit the adjusted data just as the true response function is used to fit the unadjusted instrument output. The modified response function and adjusted data are described by the equations

$$
\begin{aligned}
& f^{\prime}(t)=s(t) \otimes f(t), \\
& g^{\prime}(t)=g(t) \otimes s(t),
\end{aligned}
$$

where $f(t)$ is the true response function and $g(t)$ is the unadjusted instrument output. From equations 6 , 11,12 , and the commutative property of convolutions it therefore follows that:

$$
h(t) \otimes f^{\prime}(t)=g^{\prime}(t)
$$

This approach yields quantitative agreement between true and fitted injection lengths for all calorimeters tested here (Figure S2), confirming that the ITC-ERM accurate reproduces peak shapes for a range of cell sizes and geometries. Furthermore, $\mathrm{Ca}^{2+} /$ EDTA saturation experiments performed with the both ITC-200 and Nano ITC show essentially superimposable peak shapes over a broad range of stoichiometric volumes (up to about 50\% for both ITC-200 and Nano ITC) (Figure S3). This indicates that the assumption of instantaneous mixing holds for the ITC-200 and Nano ITC under conditions similar to those we found for the VP-ITC.

\section{DISCUSSION}

The advantages of using ITC to measure chemical reactions, particularly enzyme kinetics, have long been recognized and there is varied literature on the subject going back several decades.[4, 12, 43] Most of these studies have focused on reactions taking place over tens of minutes to avoid complications of the finite instrument response time. Here we demonstrate experimentally that accurate Michaelis-Menten parameters can be extracted from ITC peaks just tens of seconds in length. Computer simulations had previously predicted that accurate enzyme kinetic parameters could be extracted from ITC peaks on this timescale. However, to our knowledge, this had not been tested experimentally. The ITC reaction times utilized in this study are about a 20 -fold shorter than those of previous ITC enzyme kinetic studies, and thus represent an important experimental validation of the theoretical predictions.[9, 19] Rapid ITC kinetic experiments (seconds) offer several advantages compared to longer-timescale (minutes) approaches. Firstly, they allow the same number of kinetic traces to be obtained in much less time, which is advantageous both in terms of throughput, and for substrates that degrade or enzymes that steadily lose activity under catalytic conditions, such as Cytochrome P450 and NADPH-cytochrome P450 reductase.[44] Secondly, they are well suited to small injections of dilute substrates that are consumed rapidly by the enzyme, which is desirable for expensive or sparingly soluble reagents. This is also beneficial for systems that experience product inhibition, as operating at lower substrate concentrations means generating less product. Lastly, a compensation calorimeter measures reaction rates in terms of the displacement of the heat flow from the baseline value. However, the ITC baseline fluctuates slowly with time and must be interpolated between the beginning of the injection and the end of the reaction. [11, 36] Shorter reactions allow for smaller regions of interpolation and thus fewer errors due to baseline fluctuations. 
We found that a previously published (variable- $\tau$ ) approach for analyzing ITC kinetic traces yielded accurate enzyme parameters, even for short reactions on the order of tens of seconds. However, for rapid reactions (less than about 80 seconds), the inherent assumption of a mono-exponential instrument response led to large systematic distortions of the calculated ITC peaks shapes. These distortions were eliminated by replacing the mono-exponential response function with an empirical response function that is derived experimentally from very short burst injections using a rapidly-reacting host/guest system (or 1-second reference injections and suitably adjusted data, as described above). We term this the ITC-ERM approach. Most kinetic ITC studies to date have assumed mono-exponential[9, 11, 19, 20, 36] or biexponential[32, 35, 45] instrument response functions. However the empirical response generated by a short burst of heat in the calorimeter cell is not well approximated by mono-, bi-, or tri-exponential functions (Supplemental Figure 6 and 7) and good agreement is obtained with sums of about 14 exponential terms or more (Supplemental Figure 8). In principle, it would be possible to fit the experimental instrument response with a multi-exponential function, which could then be used in subsequent ITC-ERM calculations. However the experimental instrument response curves are visually extremely smooth to begin with and we did not find the additional smoothing provided by a multiexponential function to be necessary. We therefore averaged and area-normalized the experimental data for 5-10 short injections, and used these values directly in numerical convolutions to simulate ITC data.

The ability of the ITC-ERM approach to quantitatively reproduce the fine features of ITC peaks opens the door to novel investigations of biological kinetics, particularly for systems where the kinetic mechanism is not known a priori. For instance, the ITC-ERM method clearly showed that the POP enzyme deviates from Michaelis-Menten kinetics in a concentration-dependent manner. This behavior appears only at enzyme concentrations too large to study by standard spectrometric assays, and is completely obscured by the ITC peak-shape distortions produced in the variable- $\tau$ approach. In other words, the ITCERM approach offers a unique opportunity to extract detailed enzyme kinetic information inaccessible using other experimental techniques. Furthermore, ITC-ERM approach is applicable not only to enzyme kinetics but to all kinetic ITC applications. For instance, kinetics of macromolecular ligand binding and coupled folding/binding reactions can be measured by ITC.[19] Many association reactions take place in seconds or tens of seconds under ITC conditions, meaning that methods to accurately reproduce rapid kinetic ITC peak shapes are quite important for these studies.

There are several caveats to the ITC-ERM approach. Firstly, as with any ITC-based kinetics method, both the affinity and the enthalpy must be large enough to permit detection of the interaction at relatively low concentrations. For instance, we found that for the $\mathrm{Ca}^{2+} /$ EDTA interaction, $10^{5} \mathrm{M}^{-1} \mathrm{~s}^{-1}$ represents an approximate upper limit for measurable association rate constants, under the conditions of this study. This corresponds to roughly $11 \mu \mathrm{cal}$ of heat released per injection and a pseudo-first order time constant of 2 s. To achieve similar results with another system, the concentration of the injectant would have to be adjusted to yield similar heats, while the concentration in the cell would have to be adjusted to maintain association times at or slower than $2 \mathrm{~s}$. For weak interactions with low enthalpies, this may not always be possible. Secondly, pre-reaction mixing is effectively ignored in the method as implemented here. This is justified based on the excellent agreement we find for trypsin kinetic ITC peaks using this approach, as well as our observation that mixing occurs at a much faster rate than the post-reaction heat transfer and detection steps, at least with a large excess concentration of host molecules (eg. EDTA) in the cell. For injections near the stoichiometric saturation of a host or kinetic saturation of an enzyme, we might expect this approximation to break down. Finite pre-reaction mixing has previously been modelled in ITC kinetic studies as a first-order exponential process. [19] This could easily be included in the ITC-ERM approach as an additional step in calculating the instantaneous heat $(\mathrm{h}(\mathrm{t}))$. However this does not appear to be necessary to accurately model the ITC data presented here, and details of this additional calculation are beyond the scope of this work. A second caveat is that we have found that the empirical instrument response varies as a function of temperature, stirring speed, and solvent viscosity (Figures S4, 9, 10), and solution density and thermal conductivity are likely also important.[9] Thus burst injection host/guest 
experiments must be performed under conditions as identical as possible to those of the actual kinetic data set. Note that for ITC-200 and Nano ITC calorimeters, short $(\sim 1 s)$ reference injections should be performed instead of burst $(\sim 0.2 \mathrm{~s})$ injections and the instrument output adjusted prior to analysis, as described above. A temperature series of binding kinetics will require a temperature series of burst or reference injection measurements, for example. The ITC-ERM approach is consequently more costly in terms of experiment time than methods where a simplified mono exponential response function is either optimized on the fly, such as in the variable- $\tau$ method,[9] or approximated identically for all conditions. We note that in our hands, analyses using simplified mono-exponential response functions yield accurate enzymatic parameters, even when the fitted curves deviate substantially from the experimental data (as in Figure 2B). Thus when the kinetic mechanism is known a priori and only the model parameters are of interest, there are time-saving advantages to using a simplified ITC response function. However in cases where the kinetic mechanism is not known with certainty, the distortions inherent in using a simplified mono-exponential response function completely obscure the level of agreement or disagreement between any given model and the experimental data, when ITC peaks are shorter than about 150 seconds. In contrast, the ITC-ERM reproduces such short ITC peak shapes quantitatively, allowing the appropriate mechanistic model to be selected based on goodness of fit. For example, the mono-exponential variable$\tau$ approach gives poor agreement when rapid kinetic ITC traces from either trypsin or POP are analyzed according to the Michaelis-Menten mechanism. In contrast, the ITC-ERM approach shows that trypsin data follow the Michaelis-Menten model closely, while those of POP do not, and instead follow a concentration-dependent cooperative mechanism. Thus the ITC-ERM represents a new and valuable analytical tool for extracting mechanistic information from rapid timescale ITC kinetic data and shedding new light on the function of biological macromolecules.

\section{CONCLUSION}

The present study demonstrates that ordinary calorimeters are capable of measuring heat flow with subsecond precision. However, conventional ITC data analysis approaches produce systematic deviations from experimental data when modelling rapid enzyme kinetics. We have developed an ITC-ERM approach that quantitatively reproduces ITC peak shapes, providing a simple way to study rapid reaction kinetics in detail. An application of ITC-ERM to prolyl oligopeptidase uniquely identified unexpected non-Michaelis-Menten kinetics, demonstrating its utility.

\section{ASSOCIATED CONTENT}

\section{Supporting Information}

Full mathematical treatment of instrument output, rapid injections using multiple compensation calorimeters, actual injection length vs. fitted injection length for rapid injection experiments, $\mathrm{Ca}^{2+}$-EDTA saturation experiments using ITC-200 and Nano ITC, effect of stirring speed on $\mathrm{Ca}^{2+}{ }^{2}$ EDTA injections and heater pulse peak shape, summary of fitting results for injections of BAEE into Trypsin, steady state ITC enzyme kinetics experiments, empirical response function fit to mono-, bi- and multi-exponential functions, effect of viscosity and temperature on peak shape, and table of fitted parameters for fits in figure 5, binding rate simulations, enzyme kinetics simulations, statistical analysis of errors and parameter correlation coefficient calculations, parameter correlation coefficients for fitted data. (PDF)

\section{AUTHOR INFORMATION}

Corresponding Author

*E-mail: anthony.mittermaier@mcgill.ca. Fax: 514-398-3797

\section{Author Contributions}


All authors have given approval to the final version of the manuscript. J.M.D. performed the experiments, J.M.D. and A.K.M. analyzed the data. J.M.D., N.M. and A.K.M. wrote the manuscript.

\section{ACKNOWLEDGMENT}

The Authors gratefully acknowledge Dr. Nicholas Doucet and Myriam Létourneau at the INRS for use of and help with the Nano ITC.

This research was supported by the Natural Sciences and Engineering Research Council (NSERC, Canada, grant number 327028-09) and the Canadian Institutes of Health Research (CIHR-MOP-136943) J.M.D. was supported by Group de Recherche Axé sur la Structure des Proteins (GRASP) and the Drug Development Training Program (DDTP).

\section{REFERENCES}

1. Freyer, M.W. and E.A. Lewis, Isothermal Titration Calorimetry: Experimental Design, Data Analysis, and Probing Macromolecule/Ligand Binding and Kinetic Interactions. Methods in Cell Biology, 2008. Volume 84: p. 79-113.

2. Falconer, R.J., Applications of isothermal titration calorimetry - the research and technical developments from 2011 to 2015. Journal of Molecular Recognition, 2016. 29(10): p. 504-515.

3. Leavitt, S. and E. Freire, Direct measurement of protein binding energetics by isothermal titration calorimetry. Current Opinion in Structural Biology, 2001. 11(5): p. 560-566.

4. Todd, M.J. and J. Gomez, Enzyme Kinetics Determined Using Calorimetry: A General Assay for Enzyme Activity? Analytical Biochemistry, 2001. 296(2): p. 179-187.

5. Buzzell, A. and J.M. Sturtevant, A New Calorimetric Method1,2. Journal of the American Chemical Society, 1951. 73(6): p. 2454-2458.

6. Willson, R.J., et al., Determination of Thermodynamic and Kinetic Parameters from Isothermal Heat Conduction Microcalorimetry: Applications to Long-Term-Reaction Studies. Journal of Physical Chemistry, 1995. 99.

7. Olsen, S.N., Applications of isothermal titration calorimetry to measure enzyme kinetics and activity in complex solutions. Thermochimica Acta, 2006. 448(1): p. 12-18.

8. Williams, B.A. and E.J. Toone, Calorimetric evaluation of enzyme kinetic parameters. Journal of Organic Chemistry, 1993. 58(13): p. 3507-3510.

9. Demarse, N.A., et al., Determining Enzyme Kinetics via Isothermal Titration Calorimetry, in Methods in Molecular Biology. 2015.

10. Morin, P.E. and E. Freire, Direct calorimetric analysis of the enzymatic activity of yeast cytochrome c oxidase. Biochemistry, 1991. 30(34): p. 8494-8500.

11. Transtrum, M.K., L.D. Hansen, and C. Quinn, Enzyme kinetics determined by single-injection isothermal titration calorimetry. Methods, 2015. 76: p. 194-200.

12. Watt, G.D., A microcalorimetric procedure for evaluating the kinetic parameters of enzyme-catalyzed reactions: kinetic measurements of the nitrogenase system. Analytical biochemistry, 1990. 187(1): p. 141-146.

13. Karim, N. and S.-i. Kidokoro, Precise and continuous observation of cellulase-catalyzed hydrolysis of cellooligosaccharides using isothermal titration calorimetry. Thermochimica Acta, 2004. 412(1-2): p. 91-96.

14. Karim, N., H. Okada, and S.-i. Kidokoro, Calorimetric evaluation of the activity and the mechanism of cellulases for the hydrolysis of cello-oligosaccharides accompanied by the mutarotation reaction of the hydrolyzed products. Thermochimica Acta, 2005. 431(1-2): p. 9-20.

15. Poppe, H., et al., Cyclic nucleotide analogs as probes of signaling pathways. Nature Methods, 2008. 5(4): p. 277-278.

16. Poduch, E., et al., Design of Inhibitors of Orotidine Monophosphate Decarboxylase Using Bioisosteric Replacement and Determination of Inhibition Kinetics. Journal of Medicinal Chemistry, 2006. 49(16): p. 4937-4945.

17. Hansen, L.D., et al., Enzyme-catalyzed and binding reaction kinetics determined by titration calorimetry. Biochimica et Biophysica Acta (BBA) - General Subjects, 2016. 1860(5): p. 957-966.

18. Egawa, T., et al., Method for determination of association and dissociation rate constants of reversible bimolecular reactions by isothermal titration calorimeters. Analytical chemistry, 2007. 79(7): p. 2972-2978.

19. Burnouf, D., et al., kinITC: a new method for obtaining joint thermodynamic and kinetic data by isothermal titration calorimetry. Journal of the American Chemical Society, 2011. 134(1): p. 559-565.

20. Vander Meulen, K.A. and S.E. Butcher, Characterization of the kinetic and thermodynamic landscape of RNA folding using a novel application of isothermal titration calorimetry. Nucleic Acids Research, 2012. 40(5): p. $2140-2151$.

21. Bec, G., et al., Thermodynamics of HIV-1 Reverse Transcriptase in Action Elucidates the Mechanism of Action of Non-Nucleoside Inhibitors. Journal of the American Chemical Society, 2013. 135(26): p. 9743-9752.

22. Navratilova, I., et al., Thermodynamic benchmark study using Biacore technology. Analytical Biochemistry, 2007. 364(1): p. 67-77. 
23. Homola, J., S.S. Yee, and G. Gauglitz, Surface plasmon resonance sensors: review. Sensors and Actuators BChemical, 1999. 54(1-2): p. 3-15.

24. Bisswanger, H., Binding Measurements, in Practical Enzymology. 2011, Wiley-VCH Verlag GmbH \& Co. KGaA. p. 265-296.

25. Ashenafi, M., et al., Purification and Characterization of Aminoglycoside Phosphotransferase APH(6)-Id, a Streptomycin Inactivating Enzyme. Molecular and cellular biochemistry, 2014. 387(0): p. 207-216.

26. Zheng, X.W., et al., Analytical methods for kinetic studies of biological interactions: A review. Journal of Pharmaceutical and Biomedical Analysis, 2015. 113: p. 163-180.

27. Exnowitz, F., B. Meyer, and T. Hackl, NMR for direct determination of Km and Vmax of enzyme reactions based on the Lambert W function-analysis of progress curves. Biochimica et Biophysica Acta (BBA) - Proteins and Proteomics, 2012. 1824(3): p. 443-449.

28. Shaw, E., M. Mares-Guia, and W. Cohen, Evidence for an Active-Center Histidine in Trypsin through Use of a Specific Reagent, 1-Chloro-3-tosylamido-7-amino-2-heptanone, the Chloromethyl Ketone Derived from No-Tosyl-Llysine*. Biochemistry, 1965. 4(10): p. 2219-2224.

29. Mariaule, G., et al., 3-Oxo-hexahydro-1H-isoindole-4-carboxylic Acid as a Drug Chiral Bicyclic Scaffold: StructureBased Design and Preparation of Conformationally Constrained Covalent and Noncovalent Prolyl Oligopeptidase Inhibitors. Journal of Medicinal Chemistry, 2015. 59(9): p. 4221-4234.

30. Ebrahimi, K., et al., Accurate label-free reaction kinetics determination using initial rate heat measurements. Scientific reports, 2015. 5: p. 16380.

31. Wiseman, T., et al., Rapid measurement of binding constants and heats of binding using a new titration calorimeter. Analytical Biochemistry, 1989. 179(1): p. 131-137.

32. García-Fuentes, L., C. Barón, and O.L. Mayorga, Influence of Dynamic Power Compensation in an Isothermal Titration Microcalorimeter. Analytical Chemistry, 1998. 70(21): p. 4615-4623.

33. Freire, E., O.L. Mayorga, and M. Straume, Isothermal titration calorimetry. Analytical Chemistry, 1990. 62(18).

34. Lopez-Mayorga, O., P.L. Mateo, and M. Cortijo, The use of different input signals for dynamic characterisation in isothermal microcalorimetry. Journal of Physics E: Scientific Instruments, 1986. 20.

35. Velazquez-Campoy, A., O. LÛpez-Mayorga, and M.O. Cabrerizo-VÌlchez, Determination of the Rigorous Transfer Function of an Isothermal Titration Microcalorimeter With Peltier Compensation. Journal of Thermal Analysis and Calorimetry, 1999. 57.

36. Hansen, C.W., et al., Correction for instrument time constant and baseline in determination of reaction kinetics. International Journal of Chemical Kinetics, 2011. 43(2): p. 53-61.

37. Naraghi, M., T-jump study of calcium binding kinetics of calcium chelators. Cell calcium, 1997. 22(4): p. 255-268.

38. Ingami, T., The Mechanism of the Specificity of Trypsin Catalysis. Journal of Biological Chemistry, 1963. 239(2).

39. Kelly, T., Fibroblast activation protein- $\alpha$ and dipeptidyl peptidase IV (CD26): Cell-surface proteases that activate cell signaling and are potential targets for cancer therapy. Drug Resistance Updates, 2005. 8(1): p. 51-58.

40. Polgár, L., The prolyl oligopeptidase family. Cellular and Molecular Life Sciences CMLS, 2002. 59(2): p. 349-362.

41. Porter, C.M. and B.G. Miller, Cooperativity in monomeric enzymes with single ligand-binding sites. Bioorganic Chemistry, 2012. 43: p. 44-50.

42. Szeltner, Z., et al., Electrostatic Environment at the Active Site of Prolyl Oligopeptidase Is Highly Influential during Substrate Binding. Journal of Biological Chemistry, 2003. 278(49): p. 48786-48793.

43. Buzzell, A. and J.M. Strutevant, A New Calorimetric Method. Journal of the American Chemical Society, 1951. 73(6).

44. Guengerich, F.P., et al., Measurement of cytochrome P450 and NADPH-cytochrome P450 reductase. Nat. Protocols, 2009. 4(9): p. 1245-1251.

45. Lopez, M., P.L. Mateo, and M. Cortijo, The use of different input signals for dynamic characterisation in isothermal microcalorimetry. Journal of Physics E: Scientific Instruments, 1987. 20. 
